\title{
Social media attention and citations of published outputs from re-use of clinical trial data: a matched comparison with articles published in the same journals
}

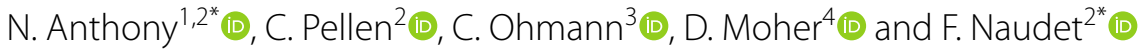

\begin{abstract}
Background: Data-sharing policies in randomized clinical trials (RCTs) should have an evaluation component. The main objective of this case-control study was to assess the impact of published re-uses of RCT data in terms of media attention (Altmetric) and citation rates.

Methods: Re-uses of RCT data published up to December 2019 (cases) were searched for by two reviewers on 3 repositories (CSDR, YODA project, and Vivli) and matched to control papers published in the same journal. The Altmetric Attention Score (primary outcome), components of this score (e.g. mention of policy sources, media attention) and the total number of citations were compared between these two groups.
\end{abstract}

Results: 89 re-uses were identified: 48 (53.9\%) secondary analyses, 34 (38.2\%) meta-analyses, 4 (4.5\%) methodological analyses and 3 (3.4\%) re-analyses. The median (interquartile range) Altmetric Attention Scores were 5.9 (1.3-22.2) for re-use and $2.8(0.3-12.3)$ for controls $(p=0.14)$. No statistical difference was found on any of the components of in the Altmetric Attention Score. The median (interquartile range) numbers of citations were $3(1-8)$ for reuses and 4 $(1-11.5)$ for controls $(p=0.30)$. Only $6 / 89$ re-uses $(6.7 \%)$ were cited in a policy source.

Conclusions: Using all available re-uses of RCT data to date from major data repositories, we were not able to demonstrate that re-uses attracted more attention than a matched sample of studies published in the same journals. Small average differences are still possible, as the sample size was limited. However matching choices have some limitations so results should be interpreted very cautiously. Also, citations by policy sources for re-uses were rare.

Trial registration: Registration: osf.io/fp62e

Keywords: Data-sharing, Data reuse, Altmetric, Individual Participant Data, Clinical trial, Scientific transparency, Reproducibility, Attention score, Research impact

\footnotetext{
*Correspondence: a-norah@hotmail.fr; floriannaudet@gmail.com

1 University Hospital of La Réunion, Saint-Denis, Reunion Island, France

${ }^{2}$ Univ Rennes, CHU Rennes, Inserm, CIC 1414 [(Centre d'Investigation

Clinique de Rennes)], F-35000 Rennes, France

Full list of author information is available at the end of the article
}

(c) The Author(s) 2021. Open Access This article is licensed under a Creative Commons Attribution 4.0 International License, which permits use, sharing, adaptation, distribution and reproduction in any medium or format, as long as you give appropriate credit to the original author(s) and the source, provide a link to the Creative Commons licence, and indicate if changes were made. The images or other third party material in this article are included in the article's Creative Commons licence, unless indicated otherwise in a credit line to the material. If material is not included in the article's Creative Commons licence and your intended use is not permitted by statutory regulation or exceeds the permitted use, you will need to obtain permission directly from the copyright holder. To view a copy of this licence, visit http://creativecommons.org/licenses/by/4.0/. The Creative Commons Public Domain Dedication waiver (http://creativeco mmons.org/publicdomain/zero/1.0/) applies to the data made available in this article, unless otherwise stated in a credit line to the data. 


\section{Key messages}

- Few re-uses have been published since the creation of the three data-sharing platforms.

- Most of them were secondary analyses or meta-analyses.

- No systematic impact of re-use was found in terms of the Altmetric Attention Score, media attention, social media attention and citation metrics however small averaged differences in favor of re-use are still plausible.

- Citations by policy sources of reuses were rare, and likewise for the controls.

- Re-uses with highest Altmetric Attention Score were mainly IPD meta-analyses

- Re-analyses were rare, however two out of the three were among the re-uses with the highest Altmetric Attention Score.

- Importantly, a high Altmetric Attention Score does not imply high scientific quality.

\section{Introduction}

In recent years, a cultural shift in research practices has occurred with the promotion of clinical trial data-sharing and reuse $[1,2]$. An increasing number of journals tend to have a data-sharing policy and encourage researchers to publish their data. Both the BMJ and PLOS Medicine even require data-sharing as a condition for publication of randomized controlled trials (RCTs). This change has taken place in a favorable scientific context. The growing movement within the scientific community to make science more open and accessible [3], as well as the awareness of a reproducibility crisis [4], are contributing to the adoption of more transparent research practices [5] that include data-sharing. Recent initiatives such as the FAIR guidelines (findable, accessible, interoperable and reusable) provide guidance on how to improve the reusability of data [1].

Sharing data generated by interventional clinical trials is considered an ethical obligation according to the International Committee of Medical Journal Editors (ICMJE) [6]. As participants have put themselves at risk in clinical trials, it is a moral obligation that the value arising from the collected data should be maximized. Besides enhancing both reproducibility and transparency, datasharing optimizes the use of limited resources (time, costs and number of patients exposed) hence reducing research wastage [7-10]. In terms of improvement of scientific knowledge, clinical trial data can set the basis for the generation of biomedical hypotheses and even hypothesis validation through the meta-analysis of Individual Participant Data (IPD). Finally, the accumulation of unbiased data on the safety and efficacy of the therapeutics being tested is crucial to better inform public health decisions, and indeed to improve the care that is offered to patients $[11,12]$.

However, this new paradigm faces barriers related to the feasibility of the requirements, the resources required, and the potential threats to trial participants [13]. Indeed, clinical trial data requires specific precautions because of the management of sensitive data, and it entails an additional workload for data-managers. Usually the sponsors are the data managers of the clinical trial data. The attitude and role of sponsors towards data-sharing can also contribute to reluctance. There is no standardization of policies across funders of clinical trials, and for those with a policy, implementation was sub-optimal [14]. Other barriers such as "fear of being wrong in public and concerns about so-called hostile reanalysis" can also limit acceptance of data-sharing practices [15].

To address these barriers, data-sharing policies should have an evaluation component to demonstrate whether the benefits outweigh the possible shortcomings. This evaluation component is currently lacking. To date, there has been no systematic attempt to assess the impact of published re-uses from clinical trials which we expected to be important, in line with the expectations of the ICMJE [6]. In a first step, an analysis of the output from data repositories can provide some useful information. These repositories, such as the YODA project [16], Clinical Study Data Request (CSDR) [17] and VIVLI [18], enable controlled access to and re-use of anonymized IPD from clinical trials. The main objective of this case-control study was therefore to assess the impact of published re-uses of data from clinical trials in terms of media attention (Altmetric) and citation rates.

\section{Method}

The protocol of the study was registered on Open Science Framework (OSF) on December 27th 2019 (registration number osf.io/fp62e). As there are no specific guidelines for meta-research studies, we have adapted the PRISMA checklist [19] as a guide to report our study.

\section{Eligibility criteria \\ Cases (Re-uses)}

All published re-uses on 3 data repositories were assessed: clinicalstudydatarequest.com (CSDR), Yale University Open Data Access (YODA), and Vivli. These data repositories were chosen because they were identified as the 3 main data repositories in a previous scoping review [20]. CSDR, funded by a consortium of clinical funders/ sponsors, seeks to be "the researcher-preferred and trusted" platform for responsible sharing of high-quality 
patient-level data for the purpose of facilitating innovative data-driven research leading to improvements in patient care. As of 31 January 2020 there was 2123 RCTs listed. The YODA project, funded by Johnson \& Johnson (formerly funded by Medtronic Inc), "advocates the responsible sharing of clinical research data, open science, and research transparency". The number of RCTs listed was 334 in July 2019. Vivli runs a data-sharing platform that includes an independent-access general data repository which is available to search for data from clinical trials conducted by researchers in academic institutions, industry, foundations and non-profit entities that can be hosted, shared and accessed. The number of RCTs listed was 4920 in March 2020. These 3 repositories, as part of their metrics, detail all published outputs related to the randomized clinical trial (RCT) data that they host [20]. Any re-use mentioned on these platforms and published in a peer review journal in English, French, German or Spanish between the creation of the platforms and $31^{\text {st }}$ December 2019 was considered. Re-uses published only as part of congress proceedings were not included nor were re-uses not listed on PubMed. The reuses were classified as secondary analyses, meta-analyses, methodological analyses or re-analyses.

\section{Controls}

Each selected publication of a re-use was matched with an article published in the same journal. In case of reuses published in specialty journals, the article listed immediately before or after the re-use in the table of contents was randomly chosen. In the absence of journals published by volume/issue (therefore absence of table of contents), online publication dates were used to choose the closest paper published before or after in the same journal. In case of re-uses published in a general medical journal, matching was performed by research field (using the International Classification of Diseases categorization: ICD-11 for Mortality and Morbidity Statistics (ICD-11 MMS), 2018 version https://icd.who.int/ browse11/l-m/en), and the closest control with matching content, randomly chosen between before and after and based on online publication date, was selected. RCT data re-uses or publications on non-human data were not considered for the formation of the control group. All controls were classified according to whether or not they were clinical trials.

\section{Primary publications}

A "primary publication" group was also set up, including publications from which the re-uses were derived. For a given re-used RCT, a "primary publication" (i.e. the main publication of the dataset that was re-used) was defined as the first article reporting fully the primary outcome of the study. In many cases (e.g. IPD meta-analyses), there were several primary publications per re-use. Also, some primary publications were related to several different re-uses. These primary publications were sought on the data-sharing platform, the clinical trial register (e.g. clinicaltrials.gov) and the manufacturer website.

\section{Data sources}

Two reviewers (NA and CP) among a team of four (NA, CP, FN, CO) independently assessed the 3 platforms. PMID (PubMed identifier) were used as identifiers for re-uses. In case of disagreement between the pair of independent reviewers, a third reviewer arbitrated. The matching of re-uses with controls and the identification of primary publications was performed by one reviewer (NA).

\section{Outcomes}

\section{Primary outcome}

The primary outcome was the Altmetric Attention Score. This score is an automatically calculated, weighted count of all of the mentions the Altmetric company has tracked for an individual research output, and is designed as an indicator of the amount and the extent of the attention an item has received [21]. The Altmetric Attention Score is mostly based on social media attention but also includes attention in newspapers and in policy sources. It is an increasingly recognized tool, which aims to measure the real-time influence and outreach of academic articles [22].

\section{Secondary outcomes}

The secondary outcomes were the citation metrics (total citations) and the following components of the Altmetric Attention Score: 1/ Policy sources (number of policy sources citing the paper), 2/ News (number of media sources that have mentioned the publication), 3/ Blogs (number of blogs that have mentioned the publication), 4/Twitter (number of the twitter accounts that have tweeted the publication), 5/ Facebook (number of the pages that have been shared on Facebook), 6/ Reddit (number of Reddit threads posted about this publication), 7/ Video (number of Youtube/Vimeo videos), 8/ Google plus (Number of accounts that have shared on Google +), 9/ Readers (Total Reader Counts, readers in citeulike count, readers in Mendeley count, readers in Connotea count).

The primary outcome and the citation metrics were assessed for the three groups (re-uses, controls, and primary publications) and the remaining secondary 
outcomes were assessed for re-uses and controls only. In the case of multiple primary publications for a re-use, the average of the quantitative outcomes among these publications was used.

\section{Data analysis}

We first performed a descriptive analysis of the general characteristics of cases and controls using basic statistical parameters (medians, first and third quartile for quantitative variables, counts and percentages for qualitative variables).

Then, the re-use and control groups were compared using the Wilcoxon rank sum test on all primary and secondary outcomes. We relied on non-parametric analyses, because of the highly skewed distribution of these outcomes, especially the Altmetric Attention Score.

As part of a secondary analysis, correlations between the Altmetric Attention Scores of the primary publications and those for re-uses, and between the number of citations of primary publications and re-uses, were explored and tested using the Spearman test. Lastly, an exploratory analysis determined whether certain types of content (Medicine (adults and children) and psychology/psychiatry (adults and children) were associated with more attention or higher citation rates. For these two groups of analysis, an adjustment by Bonferroni was performed following a comment in the peer review process.

Altmetric score-related outcomes were automatically extracted from Altmetric explorer using the R library (rAltmetric) [23]. We used the 3.4.2 version of $R$ [24]. rAltmetric retrieves data from Altmetric explorer by means of an API (application programming interface) key [25]. Agreements for the use of Altmetric data using an API key were reached with the Altmetric team on the 5th December 2019. Selection and coding were performed by four independent reviewers in independent manner.

\section{Results}

\section{Changes to the initial protocol}

In order to provide a qualitative appraisal of the impact of re-use, we added a description of the 20 reuses that attracted the most attention and the detail and context for all re-uses cited by a policy source. While we planned that two researchers would review the primary studies, only one did, given the secondary nature of the analysis and the unfavorable ratio of time spent to informativeness. Finally, two additional secondary outcomes, "score in context rank within publications in the journal" and "score in context rank within publications in this time period" were planned but not analyzed because we were no able to extract the overall number of ranks data making their interpretation impossible.

\section{Study identification}

Matching and data extraction occurred in February 2020. Data on Altmetric metrics and the number of citations were extracted on the 10th of April 2020, as part of the final analysis. Sixty-three re-uses were extracted from CSDR and 28 from YODA. There were no re-uses found on the VIVLI repository. After deletion of duplicates and matching, a total of 89 re-uses (with 197 primary RCTs identified) and 89 matched controls was obtained (Fig. 1).

\section{Description of studies included}

Re-uses from the two repositories published since 2015 were as follows 48 (53.9\%) of them were secondary analyses ( 7 were from YODA and 41 from CSDR), $34(38.2 \%)$ were meta-analyses (19 were from YODA and 15 from CSDR), 4 (4.5\%) methodological analyses (one was from YODA and 3 from CSDR) and 3 (3.4\%)

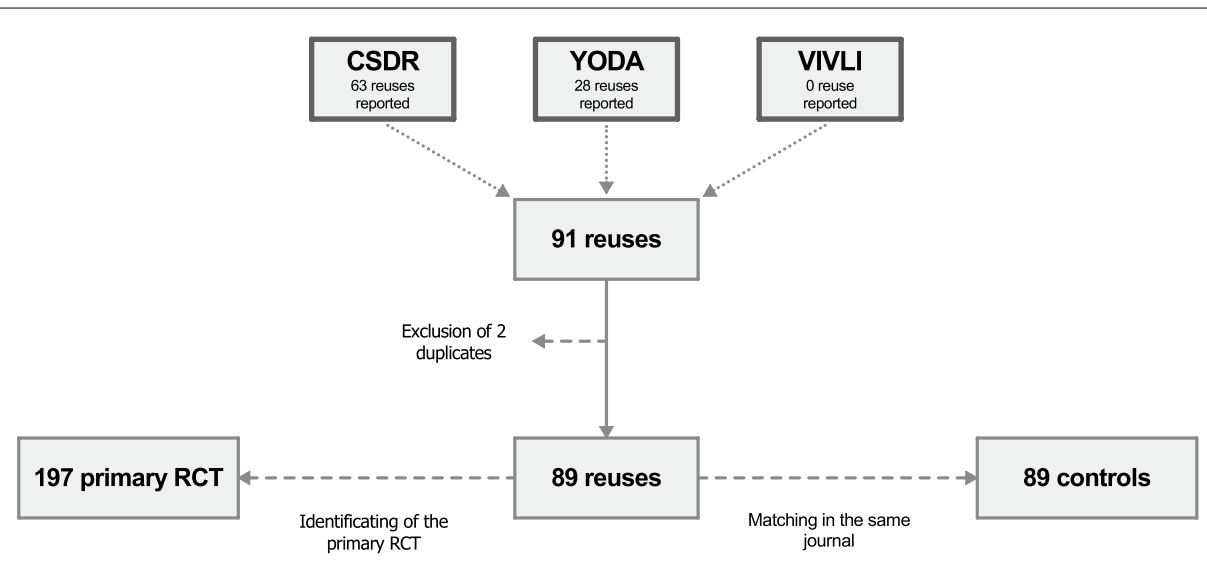

Fig. 1 Flow chart of study selection. The following figure was represented in a square root scale 
re-analyses (one was from YODA and 2 from CSDR). For the controls, 76 (85.4\%) were not clinical trials and 13 (14.6\%) were clinical trials (Web appendix 1). A total of $66(74.2 \%)$ re-uses were in the area of adult medicine, $18(20.2 \%)$ on psychology/psychiatry (children and adults) and 5 (5.6\%) on pediatrics. A total of 8 of 89 reuses were from general medical journals and required matching by ICD classification (Web appendix 2).

\section{Media attention and impact of re-use}

The median (interquartile range) Altmetric Attention Scores (Fig. 2a) were 5.9 (1.3-22.2) for re-uses and 2.8 $(0.3-12.3)$ for controls $(p=0.14)$. No statistical difference was found on the various components of the Altmetric Attention Score (Table1). Median (interquartile range) numbers of citations (Fig. 2b) were $3.0(1.0-8.0)$ for reuses and $4(1.0-11.5)$ for controls $(p=0.30)$. Detail per article type is provided in Web appendix 3. The median ( $1^{\text {st }}$ quartile, third quartile) lag between reuses and controls was $0(0,0)$.

A total of $6(6.7 \%)$ re-uses were mentioned by a policy source (Table 2): 2 meta-analyses [26, 27], 3 secondary analyses [28-30] and one re-analysis [31]. One metaanalysis and one secondary analysis were cited in "immunological basis for immunization series" of the World Health Organization (WHO). The other meta-analysis was cited in the National Institute for Health Care Excellence (NICE) guidelines as a reference for psoriatic arthritis following inadequate response to disease-modifying anti-rheumatic drugs, and its economic evaluation. Another secondary analysis was identified by Altmetric as cited by a NICE evidence review, but after careful evaluation of the NICE reviews, it appeared that this study was cited as an excluded study and therefore had no practical impact. The last secondary analysis was cited in a national immunisation programme in the Netherlands. The re-analysis was cited as an example demonstrating the need for Open Data.

The top 20 re-uses with the highest Altmetric Attention Scores ranging from 23.5 to 1407.5 are presented in Table 3: 12 (60\%) were in fields of medicine, 7 (35\%) in psychology/psychiatry and one in pediatrics (5\%). Among re-uses, 11 (55\%) were IPD meta-analyses, 5 (25\%) were secondary analyses, $2(10 \%)$ were methodological papers and $2(10 \%)$ were re-analyses.

A significant negative correlation between primary studies and re-uses for the Altmetric Attention Score was found ( $\rho=-0.31, p=0.006$, Web appendix 4) and no correlation was found for citations rates $(\rho=-0.20$, $p=0.08$, Web appendix 4). Psychiatry/psychology was associated with a higher Altmetric Attention Score than medicine (8.4 (2.3-30.9) for psychiatry/psychology and $3.2(0.3-12.1)$ for medicine; $p=0.003)$. Total citations were not found to differ between these two subgroups (5 (1.8-13.3) for psychiatry/psychology and 3 (1.0-8.8) for medicine; $p=0.11)$. There was no difference in terms of Altmetric Attention Scores nor for citations in the subgroups of Psychiatry/psychology (respectively $p=0.40$ and $p=0.69$ ) or in medicine (respectively $p=0.24$ and $p=0.38$ ). Using Bonferroni's method for multiple comparisons, both the correlation between primary studies and re-uses for the Altmetric Attention Score and the association between psychiatry/psychology content and higher Altmetric Attention Score remained significant over the 8 former correlations/comparisons $(p<0.0065)$. We did not test any interaction because the data was highly skewed and precluded the use of parametric models. All these analyses are detailed in Web Appendix 5.

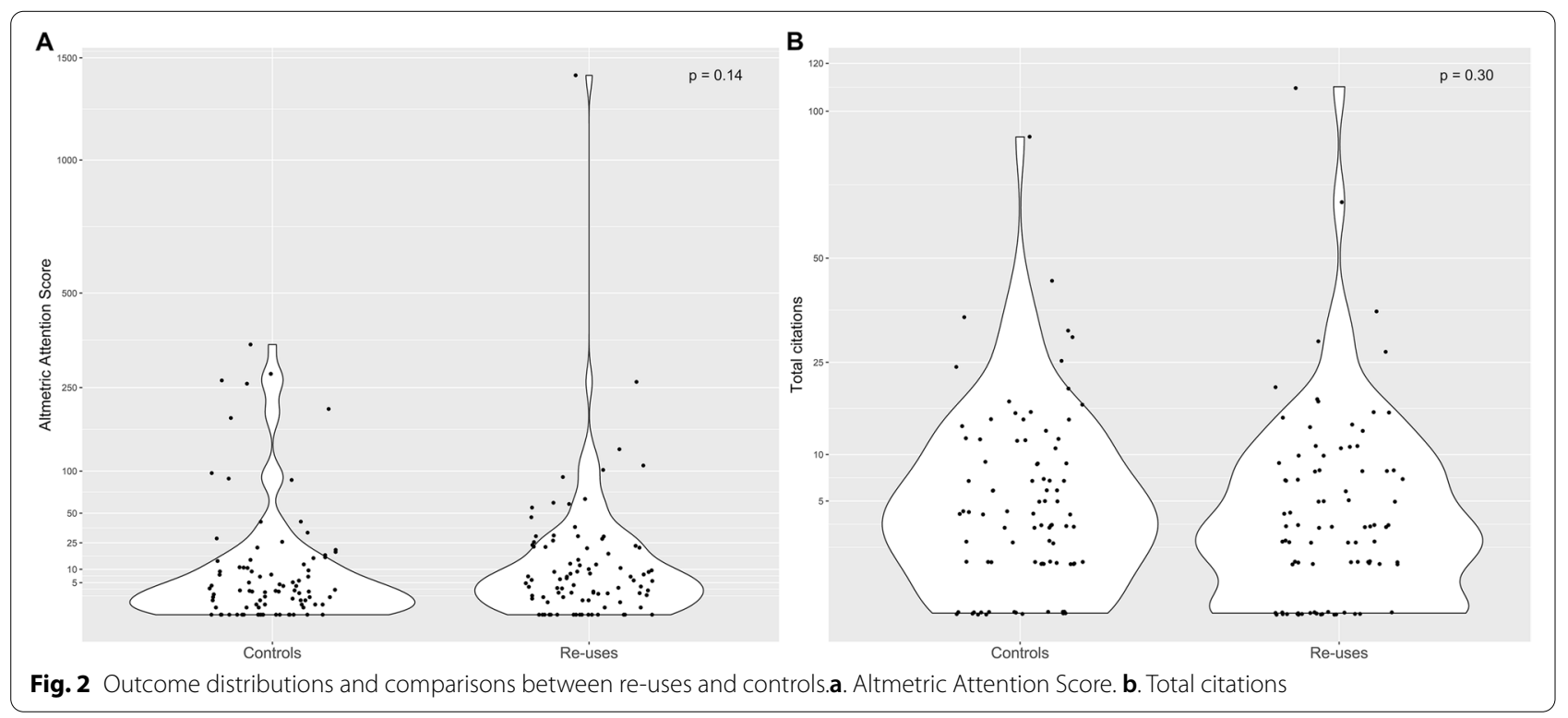


Table 1 Altmetric data for reuses and controls

\begin{tabular}{|c|c|c|c|c|}
\hline & Control $(N=89)$ & Re-use $(N=89)$ & Total $(N=178)$ & $p$-value \\
\hline Altmetric Attention Score & $2.8(0.3-12.3)$ & $5.9(1.3-22.2)$ & $3.9(0.5-15.9)$ & 0.14 \\
\hline Number of citations ${ }^{a}$ & $4(1-11.5)$ & $3(1-8)$ & $3(1-9)$ & 0.30 \\
\hline Number of policy sources that have mentioned the publication & $0(0-0)$ & $0(0-0)$ & $0(0-0)$ & 0.52 \\
\hline Number of the news sources that have mentioned the publication & $0(0-0)$ & $0(0-1)$ & $0(0-0.8)$ & 0.64 \\
\hline Number of blogs that have mentioned the publication & $0(0-0)$ & $0(0-0)$ & $0(0-0)$ & 0.17 \\
\hline Number of the Twitter accounts that have tweeted this publication & $4(1-10)$ & $5(1-17)$ & $4(1-13.75)$ & 0.14 \\
\hline Number of pages shared on Facebook & $0(0-1)$ & $0(0-1)$ & $0(0-1)$ & 0.74 \\
\hline Number of Reddit threads posted about this publication & $0(0-0)$ & $0(0-0)$ & $0(0-0)$ & 0.17 \\
\hline Number of the Youtube/Nimeo channels & $0(0-0)$ & $0(0-0)$ & $0(0-0)$ & 1 \\
\hline Number of the accounts that have shared on Google + & $0(0-0)$ & $0(0-0)$ & $0(0-0)$ & 0.40 \\
\hline Total Reader Counts & $15(2-36)$ & $15(4-31)$ & $15(3.3-35.3)$ & 0.91 \\
\hline Readers in citeulike & $0(0-0)$ & $0(0-0)$ & $0(0-0)$ & 0.32 \\
\hline Readers in Mendeley & $15(2-36)$ & $15(4-31)$ & $15(3.3-35.3)$ & 0.90 \\
\hline Readers in Connotea & $0(0-0)$ & $0(0-0)$ & $0(0-0)$ & / \\
\hline
\end{tabular}

For all quantitative outcomes, data are presented as median (interquartile range)

a 5 missing data ( 2 for controls and 3 for re-uses)

\section{Discussion}

\section{Statement of principal findings}

Using all available re-uses of clinical trial data to date from two major data repositories, we were not able to demonstrate that re-uses of clinical trial data attracted more attention than a matched sample of studies published in the same journals: while the Altmetric Attention Score was numerically higher for re-uses than for controls, the difference did not reach statistical significance, perhaps because of lack of power. The magnitude of the difference was nevertheless quantitatively small. It is important to stress that whether for re-uses or for controls, a large proportion of articles had a null or low Altmetric Attention Score. In addition, a large part of the Altmetric Attention Score was driven by activity on Twitter and only six out of 89 re-uses were cited in policy sources, with 5 out of these 6 having directly impacted these documents. Unsurprisingly most of the re-uses that attracted attention were IPD meta-analyses, as these studies present in general high-quality evidence and can attract more interest. Interestingly, 2 of the 3 reanalyses were among the re-uses that attracted the most attention. The first one [31] was the first study within the RIAT initiative (Restoring Invisible and Abandoned Trials) [32] which contradicted a previous, well known, misreported trial on paroxetine among adolescents with major depressive disorder. The second one [33] set out to reproduce SMART-AF [34], a multicenter, single-arm trial evaluating the effectiveness and safety of an irrigated, contact force-sensing catheter for ablation of drugrefractory, symptomatic, paroxysmal atrial fibrillation. This re-analysis arrived at the same general conclusions as the initial study. Attention towards re-analyses can be expected, since reproduction analyses are an important outcome from data-sharing. This being said, re-analyses were not commonly performed in the area of medicine [35] and were indeed a small portion of the re-uses we identified.

\section{Findings in relation to other studies}

Up to the present day, evidence on the impact of research output from sharing IPD from clinical trials is still very sparse. In an assessment of citation metrics for 224 reuses of clinical trial data from the NHLBI Data Repository, half of the publications based on clinical trial data had cumulative citations that ranked in the top 34\%, normalized for subject matter and year of publication. No significant difference was found compared to the percentage of $28.3 \%$ for publications based on observational studies and $29 \%$ for a $10 \%$-random sample of all NHLBIsupported articles published during the same period [36].

\section{Limitations of this study}

In this study, we explored certain indicators of impact resulting from the published output from data re-use. It should be not conflated with the impact of data-sharing. Intention to share data may not result in effective data sharing, effective data sharing may not result in effective re-use, effective re-use may not result in a published output.

Importantly, it is worth noting that both citation metrics and Altmetric data are merely a surrogate for real research impact and interest; both scores have been identified as being correlated [37-41]. While the Altmetric Attention Score has been used in previous research [42] and seems to be an acceptable substitute for media 


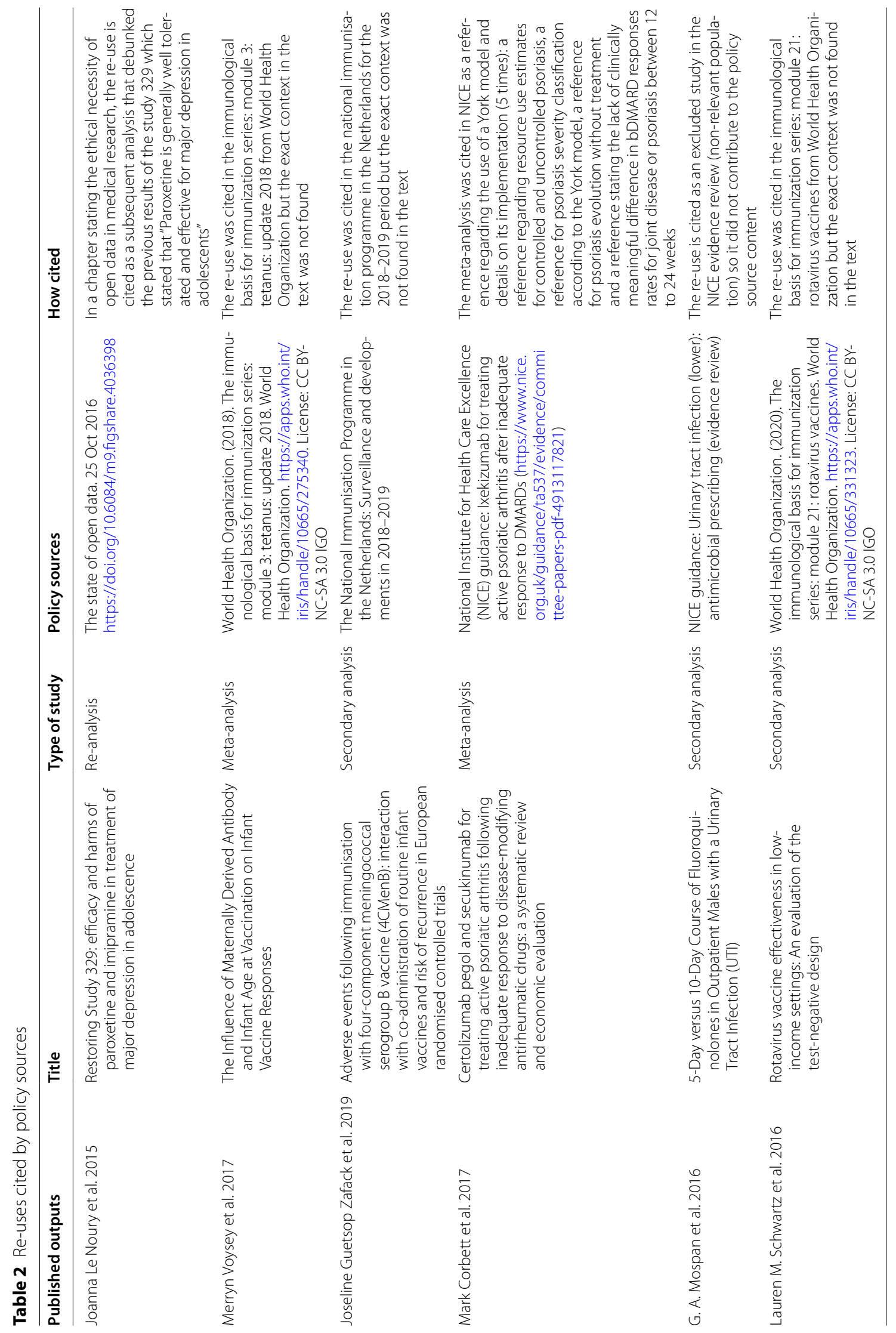




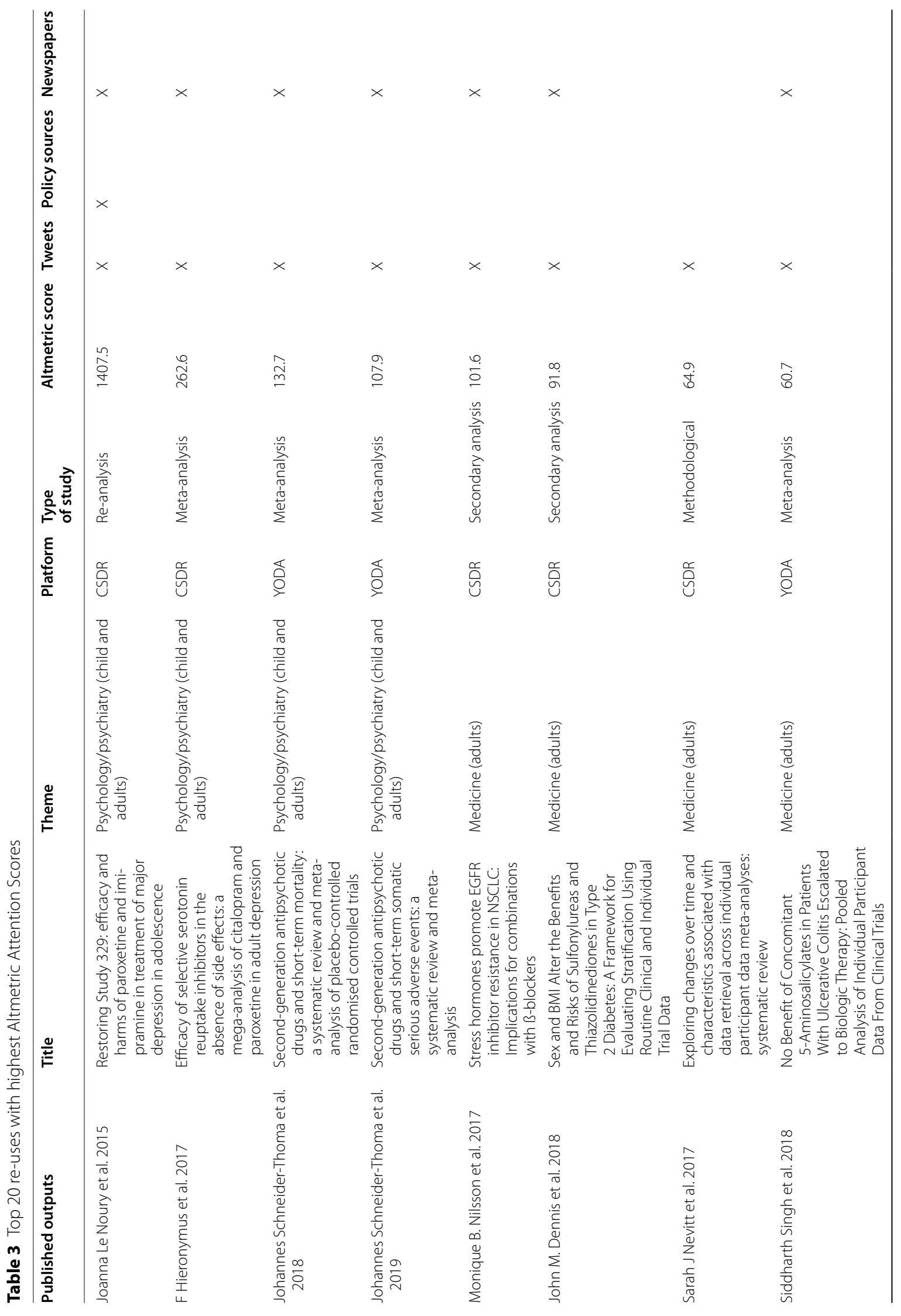




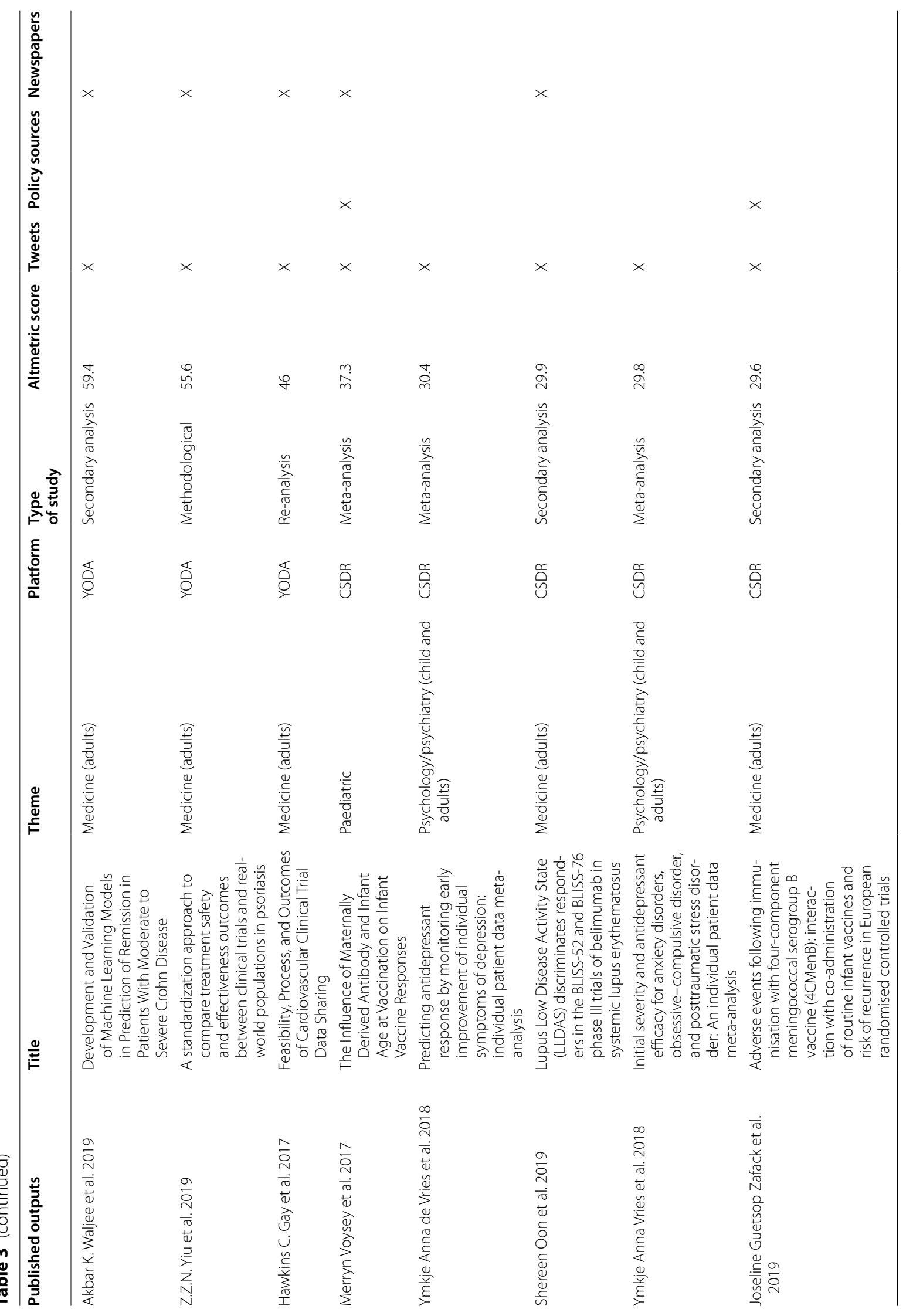




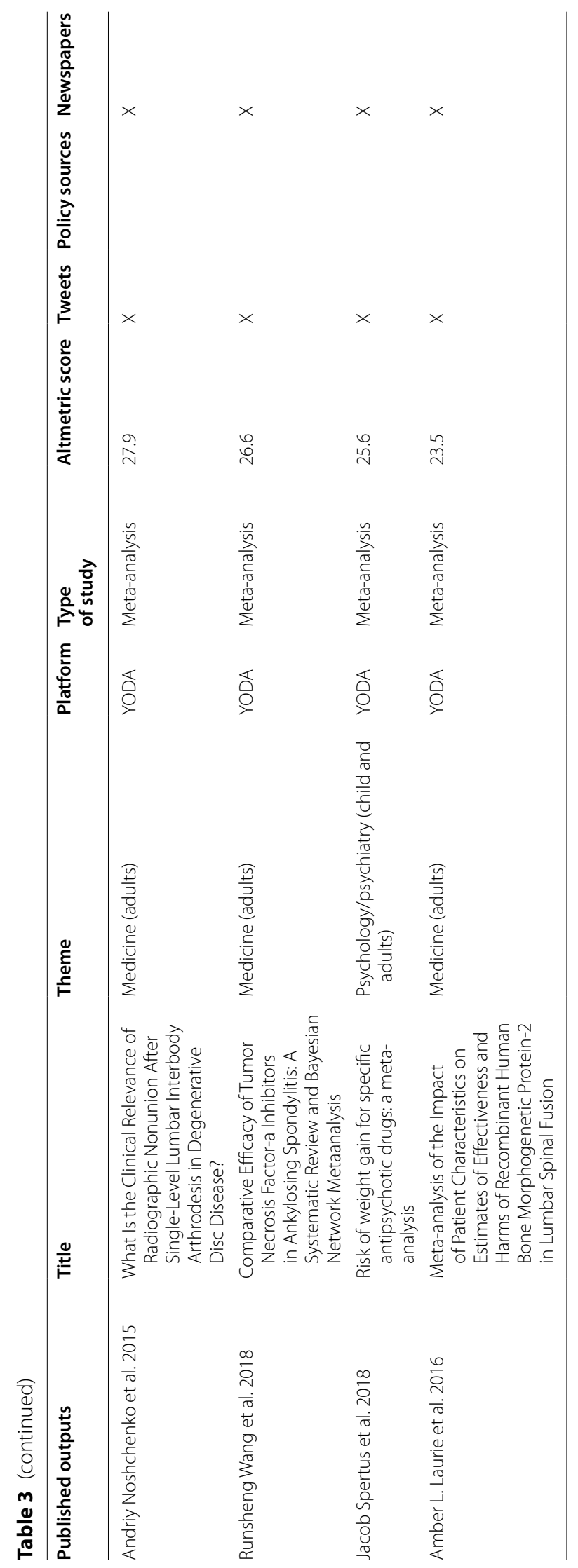


attention and a relevant indicator for measuring interest and implication by the public in scientific research, it should obviously not be confused with scientific importance. Plus, Altmetric is highly gameable for some of their aspects such as tweets. It is however way more difficult to game with certain components such as guidelines. To provide a more comprehensive picture, we therefore described qualitatively how the different re-uses were cited by policy documents. Despite being increasingly used by journals, the construction of the Altmetric Attention Score with different weights attributed to altmetrics [43] is somewhat debatable and it is unknown how much this could impact our findings. It is uncertain whether the AAS information provided is complete. Similarly, its use as a composite criterion can create interpretation issues. Therefore we have detailed all its different dimensions one by one. The low coverage of all altmetrics, except for Twitter, was highlighted in a 2013 study [44]. It is possible that the Altmetric assessment might have missed some policy sources resulting in an underestimation of the true impact of these re-uses.

In addition, while we relied on an exhaustive review of various data repositories, there were still few re-uses included in our study. And indeed, while the repositories host a large volume of clinical trial data, they are still under-used with a small number of data requests in comparison to the volume of available data [20]. This could have resulted in limited power for our study, as we did not calculate the sample size prior to the study, making it difficult to detect a small difference between the 2 groups. In addition, any comparison between groups can be affected by confounders (e.g. research field, year of publication). We tried to control for these biases using a matched control group based on the same journal, but the matching criteria can be criticized, and unmeasured or residual confounders may still exist. Also, clinical trials were rare in the control group which could have an impact on the results, as it is likely that clinical trials attract more attention than other research designs, such as for instance observational studies. However this assumption does not seem to hold true in every field; in fact a review of the 50 most cited articles in the field of pediatrics showed that they were mainly observational studies [45]. On the other hand, a re-use of clinical trial data is not exactly the same as the primary use of this data. The results of the comparisons should therefore be interpreted cautiously and it is possible that the descriptive/qualitative analysis could be much more instructive than the comparison. Also finding an "unquestionable equivalent" for a secondary analysis, a methodological analysis or a re-analysis, which is not itself a re-use of clinical trial data, was near impossible. Even if an alternative matching would have been possible, the adoption of different definitions might have implied to select controls published much sooner or much later than the re-use. It means that any improvement in a sense may make matching worst in a second sense. It could have added much more, heterogeneity and, perhaps some confounding. It is indeed expected that the publication date can influence the amount of impact received. For example, matching an IPD meta-analysis with a metaanalysis on aggregated data in the same issue of a journal would have been quite unlikely. Confounding by time of publication, could also explain the negative correlation identified between primary papers and re-uses, as the importance of earlier papers could be underestimated by some social media (e.g. Twitter) that are relatively recent. Lastly, while a total of 89 re-uses of clinical trial data was identified, additional re-uses of data that did not result in publications are likely, meaning that the (somewhat low) impact of re-use described in this paper could be an overestimation compared with the true impact of data-sharing. Conversely it is also possible that some re-uses were not yet documented on the platforms at the time of the extraction and we did not include re-uses of clinical trial data that were not shared on the 3 data-sharing platforms. Data-sharing could also occur between teams, upon request, without the use of platforms.

\section{Conclusion}

Data-sharing policies are coming progressively into effect with the aim of reforming the way clinical research is performed. However, since high-quality policies need to be evidence-based, it is necessary to assess whether they achieve the intended effects. In this perspective, and as a first step, this study documented the digital impact of published re-uses. We are however at an early stage in these policies and updating the data in the future with more knowledge and with additional altmetrics seems relevant, given the increasing proportion of re-uses [20]. In addition, adequate and reliable markers of impact and indeed usefulness [46] of these re-uses need to be developed, since neither altmetrics nor citation rates are a panacea for assessing impact.

Bearing in mind the numerous limitations of our study, we were not able to document a systematic impact of all re-uses in terms of media attention, nor in terms of use in policy documents. Still, the data collected in this study can inform future studies exploring the impact of clinical trial data-sharing. The need to modify some elements of the initial protocol may also feed the methodology of future research on this theme. This is an imperative to optimize current data-sharing practices. 


\section{Supplementary Information}

The online version contains supplementary material available at https://doi. org/10.1186/s12874-021-01311-z.

\section{Additional file 1. \\ Additional file 2. \\ Additional file 3. \\ Additional file 4. \\ Additional file 5 .}

\section{Acknowledgements}

The authors wish to thank Altmetric for providing this study data free of charge for research purposes.

\section{Authors' contributions}

$\mathrm{NA}$ and FN conceptualized the research and they both wrote the manuscript. NA analyzed data and interpreted data supervised by FN. NA and CP extracted data. NA, FN, CP, DM and CO read and approved the final manuscript.

\section{Funding}

No specific funding for this review.

FN's work on data sharing is supported by a grant from the French National Research Agency - ANR [Reproducibility in Therapeutic Research / RelTheR: ANR-17-CE36-0010-01].

\section{Availability of data and materials}

The datasets generated and/or analyzed during the current study are available in the osf.io/fp62e repository.

\section{Declarations}

\section{Ethics approval and consent to participate}

Not applicable. The study does not involve human participants.

\section{Consent for publication}

Not applicable.

\section{Competing interests}

The authors declare that they have no competing interests.

\section{Author details}

${ }^{1}$ University Hospital of La Réunion, Saint-Denis, Reunion Island, France. ${ }^{2}$ Univ Rennes, CHU Rennes, Inserm, CIC 1414 [(Centre d'Investigation Clinique de Rennes)], F-35000 Rennes, France. ${ }^{3}$ European Clinical Research Infrastructure Network, Düsseldorf, Germany. ${ }^{4}$ Ottawa Hospital Research Institute, Ottawa, Canada.

Received: 30 January 2021 Accepted: 30 April 2021

Published online: 06 June 2021

\section{References}

1. Wilkinson MD, Dumontier M, Aalbersberg ljJ, Appleton G, Axton M, Baak $A$, et al. The FAIR guiding principles for scientific data management and stewardship. Sci Data. 2016;3:160018.

2. Zarin DA, Tse T. Medicine. Moving toward transparency of clinical trials. Science. 2008:319:1340-2

3. Nosek BA, Alter G, Banks GC, Borsboom D, Bowman SD, Breckler SJ, et al. Scientific Standards promoting an open research culture. Science. 2015;348:1422-5.

4. Baker M. 1,500 scientists lift the lid on reproducibility. Nat News. 2016;533:452.

5. Bakken S. The journey to transparency, reproducibility, and replicability. J Am Med Inform Assoc JAMIA. 2019;26:185-7.
6. Taichman DB, Backus J, Baethge C, Bauchner H, de Leeuw PW, Drazen JM, et al. Sharing clinical trial data: a proposal from the international committee of medical journal editors. PLoS Med. 2016;13:e1001950.

7. Bhopal RS. Increasing value and reducing waste in biomedical research. Lancet Lond Engl. 2016;388:562.

8. Ioannidis JPA, Greenland S, Hlatky MA, Khoury MJ, Macleod MR, Moher D, et al. Increasing value and reducing waste in research design, conduct, and analysis. Lancet Lond Engl. 2014;383:166-75.

9. Glasziou P, Altman DG, Bossuyt P, Boutron I, Clarke M, Julious S, et al. Reducing waste from incomplete or unusable reports of biomedical research. Lancet Lond Engl. 2014;383:267-76.

10. Chan A-W, Song F, Vickers A, Jefferson T, Dickersin K, Gøtzsche PC, et al. Increasing value and reducing waste: addressing inaccessible research. Lancet Lond Engl. 2014;383:257-66.

11. Ohmann C, Banzi R, Canham S, Battaglia S, Matei M, Ariyo C, et al. Sharing and reuse of individual participant data from clinical trials: principles and recommendations. BMJ Open. 2017;7:e018647.

12. Lo B. Sharing clinical trial data: maximizing benefits, minimizing risk. JAMA. 2015;313:793-4

13. Taichman DB, Sahni P, Pinborg A, Peiperl L, Laine C, James A, et al. Data sharing statements for clinical trials: a requirement of the international committee of medical journal editors. Rev Med Chil. 2017;145:691-3.

14. Gaba JF, Siebert M, Dupuy A, Moher D, Naudet F. Funders' data-sharing policies in therapeutic research: a survey of commercial and noncommercial funders. PLoS ONE [Internet]. 2020 [cited 2020 Oct 11];15. Available from: https://www.ncbi.nlm.nih.gov/pmc/articles/PMC74 46799/

15. Bell V. Open science in mental health research. Lancet Psychiatry. 2017:4:525-6.

16. The YODA Project | Welcome to the YODA Project [Internet]. [cited 2020 Mar 19]. Available from: https://yoda.yale.edu/welcome-yoda-project

17. ClinicalStudyDataRequest.com [Internet]. [cited 2020 Mar 19]. Available from: https://www.clinicalstudydatarequest.com/

18. Vivli - Center for Global Clinical Research Data [Internet]. Vivli. [cited 2020 Mar 19]. Available from: https://vivli.org/

19. Liberati A, Altman DG, Tetzlaff J, Mulrow C, Gøtzsche PC, loannidis JPA, et al. The PRISMA statement for reporting systematic reviews and meta-analyses of studies that evaluate health care interventions: explanation and elaboration. Ann Intern Med. 2009;151:W65-94.

20. Ohmann C, Moher D, Siebert M, Motschall E, Naudet F. Status, use and impact of sharing individual participant data from clinical trials: a scoping review. In prep.

21. The Altmetric score is now the Altmetric Attention Score [Internet]. Altmetric. 2016 [cited 2020 Mar 19]. Available from: https://www. altmetric.com/blog/the-altmetric-score-is-now-the-altmetric-atten tion-score/

22. Warren HR, Raison N, Dasgupta P. The Rise of Altmetrics. JAMA. 2017;317:131-2.

23. Ram K. rAltmetric: Retrieves altmerics data for any published paper from "Altmetric.com" [Internet]. 2017 [cited 2020 Mar 19]. Available from: https://CRAN.R-project.org/package=rAltmetric

24. R-3.4.2 for Windows. The R-project for statistical computing. [Internet]. [cited 2020 Mar 19]. Available from: https://cran.r-project.org/bin/windo ws/base/old/3.4.2/

25. Altmetric. Altmetric Details Page API Reference \& Getting Started [Internet]. Available from: https://api.altmetric.com/

26. Voysey M, Kelly DF, Fanshawe TR, Sadarangani M, O'Brien KL, Perera R, et al. The Influence of Maternally Derived Antibody and Infant Age at Vaccination on Infant Vaccine Responses: An Individual Participant Metaanalysis. JAMA Pediatr. 2017;171:637-46.

27. Corbett M, Chehadah F, Biswas M, Moe-Byrne T, Palmer S, Soares M, et al. Certolizumab pegol and secukinumab for treating active psoriatic arthritis following inadequate response to disease-modifying antirheumatic drugs: a systematic review and economic evaluation. Health Technol Assess Winch Engl. 2017;21:1-326.

28. Mospan GA, Wargo KA. 5-day versus 10-day course of fluoroquinolones in outpatient males with a urinary tract infection (UTI). J Am Board Fam Med JABFM. 2016:29:654-62.

29. Zafack JG, Bureau A, Skowronski DM, De Serres G. Adverse events following immunisation with four-component meningococcal serogroup 
$B$ vaccine (4CMenB): interaction with co-administration of routine infant vaccines and risk of recurrence in European randomised controlled trials. BMJ Open. 2019;9:e026953.

30. Schwartz LM, Halloran ME, Rowhani-Rahbar A, Neuzil KM, Victor JC. Rotavirus vaccine effectiveness in low-income settings: An evaluation of the test-negative design. Vaccine. 2017;35:184-90.

31. Le Noury J, Nardo JM, Healy D, Jureidini J, Raven M, Tufanaru C, et al. Restoring Study 329: efficacy and harms of paroxetine and imipramine in treatment of major depression in adolescence. BMJ. 2015:351:h4320.

32. Doshi P, Dickersin $K$, Healy D, Vedula SS, Jefferson T. Restoring invisible and abandoned trials: a call for people to publish the findings. BMJ. 2013;346:f2865

33. Gay HC, Baldridge AS, Huffman MD. Feasibility, process, and outcomes of cardiovascular clinical trial data sharing: a reproduction analysis of the SMART-AF trial. JAMA Cardiol. 2017;2:1375-9.

34. Natale A, Reddy VY, Monir G, Wilber DJ, Lindsay BD, McElderry HT, et al. Paroxysmal AF catheter ablation with a contact force sensing catheter: results of the prospective, multicenter SMART-AF trial. J Am Coll Cardiol. 2014;64:647-56.

35. Ebrahim S, Sohani ZN, Montoya L, Agarwal A, Thorlund K, Mills EJ, et al. Reanalyses of randomized clinical trial data. JAMA. 2014;312:1024-32.

36. Use of the national heart, lung, and blood institute data repository | NEJM [Internet]. [cited 2020 Mar 19]. Available from: https://www.nejm.org/doi/ full/https://doi.org/10.1056/NEJMsa1603542

37. Barakat AF, Nimri N, Shokr M, Mahtta D, Mansoor H, Masri A, et al. Correlation of altmetric attention score and citations for high-impact general medicine journals: a cross-sectional study. J Gen Intern Med. 2019;34:825-7.

38. Warren VT, Patel B, Boyd CJ. Analyzing the relationship between altmetric score and literature citations in the implantology literature. Clin Implant Dent Relat Res. 2020;22:54-8.
39. Rong LQ, Lopes AJ, Hameed I, Gaudino M, Charlson ME. Examining the correlation between altmetric score and citation count in the anaesthesiology literature. Br J Anaesth. 2020;125:e223-6.

40. Mullins $\mathrm{CH}$, Boyd CJ, Corey BL. Examining the correlation between altmetric score and citations in the general surgery literature. J Surg Res Elsevier. 2020;248:159-64.

41. Huang W, Wang P, Wu Q. A correlation comparison between altmetric attention Scores and citations for six PLOS journals. PLOS ONE. Public Library of Science; 2018;13:e0194962.

42. Grundy Q, Dunn AG, Bourgeois FT, Coiera E, Bero L. Prevalence of disclosed conflicts of interest in biomedical research and associations with journal impact factors and altmetric scores. JAMA. 2018;319:408.

43. How is the Altmetric Attention Score calculated? [Internet]. Metr. [cited 2020 Mar 19]. Available from: https://help.altmetric.com/support/solut ions/articles/6000060969-how-is-the-altmetric-attention-score-calcu lated-

44. Thelwall M, Haustein S, Larivière V, Sugimoto CR. Do altmetrics work? Twitter and ten other social web services. PLoS ONE. 2013:8:e64841.

45. Jelicic Kadic A, Kovacevic T, Runjic E, Simicic Majce A, Markic J, Polic B, et al. Research methodology used in the 50 most cited articles in the field of pediatrics: types of studies that become citation classics. BMC Med Res Methodol. 2020;20:60

46. Ioannidis JPA. Why Most Clinical Research Is Not Useful. PLoS Med. 2016;13:e1002049.

\section{Publisher's Note}

Springer Nature remains neutral with regard to jurisdictional claims in published maps and institutional affiliations.
Ready to submit your research? Choose BMC and benefit from:

- fast, convenient online submission

- thorough peer review by experienced researchers in your field

- rapid publication on acceptance

- support for research data, including large and complex data types

- gold Open Access which fosters wider collaboration and increased citations

- maximum visibility for your research: over $100 \mathrm{M}$ website views per year

At BMC, research is always in progress.

Learn more biomedcentral.com/submissions 